\title{
Models of financial markets with extensive participation incentives
}

\author{
C. H. Yeung, ${ }^{1}$ K. Y. Michael Wong, ${ }^{1}$ and Y.-C. Zhang ${ }^{1,2}$ \\ ${ }^{1}$ Department of Physics, The Hong Kong University of Science and Technology, Hong Kong, China \\ ${ }^{2}$ Département de Physique, Université de Fribourg, Pérolles, Fribourg, CH-1700 Switzerland
}

(Received 31 July 2007; published 14 February 2008)

\begin{abstract}
We consider models of financial markets in which all parties involved find incentives to participate. Strategies are evaluated directly by their virtual wealth. By tuning the price sensitivity and market impact, a phase diagram with several attractor behaviors resembling those of real markets emerge, reflecting the roles played by the arbitrageurs and trendsetters, and including a phase with irregular price trends and positive sums. The positive sumness of the players' wealth provides participation incentives for them. Evolution and the bid-ask spread provide mechanisms for the gain in wealth of both the players and market makers. New players survive in the market if the evolutionary rate is sufficiently slow. We test the applicability of the model on real Hang Seng Index data over 20 years. Comparisons with other models show that our model has a superior average performance when applied to real financial data.
\end{abstract}

DOI: 10.1103/PhysRevE.77.026107

PACS number(s): 89.65.Gh, 02.50.Le, 05.40.-a

\section{INTRODUCTION}

The potential of using agent-based models to analyze and elucidate the behavior of financial markets has been gradually realized in the physics community in recent years [1]. These models are able to relate the attributes of the individual players, such as their memory sizes and payoffs, to the collective behavior of the system, such as the information content, volatility, and phase transitions [2-5]. The key insight of a family of these models, known as the minority games, is the observation that the players making the minority decisions take advantage of their counterparts during trading, and the adaptive behavior towards this target results in the nontrivial behavior of the financial markets.

However, the original minority game does not provide a correct perspective to model financial markets, in which players participate only when they see chances of getting profits. Since the winners in the games belong to the minority group at each time step, they become negative-sum games. Consequently, were the players given the option to withdraw from the market, their participation would not be sustained.

Variations of the original version of the game provided partial solutions to the issue. For example, the influence of wealth in the decisions of the agents was considered [6-8]. In these models, the buying power of an agent is curtailed when she does not have enough wealth. This creates a natural mechanism to expel the poor agents from the market, although the issue of participation incentives was not the focus of those studies.

Another mechanism for the agents to change their status of participation was considered in the so-called grandcanonical minority game [6-9]. The speculators have the option to participate or withdraw from the market according to their perception of profitability. However, the wealth gained by the so-called producers, whose predictable strategies made them the prey of arbitrage by the speculators, is at best zero. Thus, the issue merely shifts from the speculators to the producers: were the producers given the option to withdraw from the market, their participation would not be sustained.
The \$-game considered whether the market makers can be prevented from being arbitraged by the players [5]. It was found that the market makers are able to arbitrage the players when the strategies of the players are too complex, but are arbitraged by the players otherwise. The issue of voluntary participation remains: either the market makers or the players are tempted to withdraw from the game, given the option.

The agent-based models are remote from realistic financial markets in another aspect. More often, agents in real markets evaluate their strategies in real financial terms, namely, how much wealth the strategies would have brought them in the market history, had the players adopted them. In contrast, players in the agent-based models use various ways to update the virtual points or scores of their strategies when they make transactions. Typical virtual point updating rules, such as those in the original minority game [2], evaluate the buying and selling decisions at a time step, regardless of the need to update the historical effects of the previous decisions. In other models, one-step expectations of the players are considered, leading to the \$-game [5], and markets with a mixture of majority and minority game players [10]. However, these models of myopic players may not reflect the history-dependent considerations of real market players.

An example is the evaluation of holding a position (that is, a decision of taking no buying or selling actions). In real markets, players need the option to hold a position, either by actively including it as a decision prescribed by their strategies, or by passively refraining from buying and selling decisions, consequential to their reaching maximum or minimum positions, respectively. Virtual point updates in most agent-based models are neutral to holding positions.

To incorporate the option of holding a position, the most common method used in the grand-canonical models is to monitor whether the virtual points passively fall below a certain threshold $[4,9]$. Now if holding a position is part of a player's option, the benefits of taking such a position should be reflected in the payoff functions, so that the players can choose to hold long (short) positions when prices are rising (falling). These considerations are best reflected in monitoring the wealth associated with the strategy decisions. We therefore focus on wealth-based strategies in this paper. 
Wealth-based strategies were previously considered in exogenous markets [11]. As will be shown, agents with wealthbased strategies in endogenous markets can self-organize to exhibit even more interesting coordinated behaviors.

In this paper, we consider models of financial markets in which all parties involved find incentives to participate. Our models incorporate several realistic features recently added to the minority game, including wealth-based strategies and the opening and closing of an agent's position during trading $[11,12]$, but the focus will be the issue of participation incentives, attractor behavior, and tests with real data. As will be shown, the players are able to arbitrage the market makers in the absence of the spread in the bid and ask prices, provided that the market impact or the price sensitivity to the excess demand is sufficiently low. On the other hand, in an evolving market, underperforming players leave the market and new players bring their wealth into the market, rendering it a positive-sum game. The market makers share the wealth by imposing spreads in the bid-ask prices. To complete the picture, we find that it is possible for the new players to have incentives to participate if the evolutionary rate is sufficiently slow. Compared with previous models, our models are relatively simple in terms of the number of parameters, and may be eventually amenable to analytical approaches similar to those which have proved successful in the study of the minority game [13-15].

The paper is organized as follows. After introducing the model in Sec. II, we describe in Sec. III the different attractor behaviors resembling those in real markets and map the positive sumness of the players in the absence evolution and the bid-ask spread. In Sec. IV we consider the effects of the bid-ask spread imposed by the market makers. In Sec. V, we complete the picture by considering the conditions under which the new players in an evolving market are able to enhance their survival. In Sec. VI, we test the applicability of wealth-based strategies on real Hang Seng index data, followed by a conclusion in Sec. VII.

\section{MODEL}

We consider a model of the financial market in which $N$ agents trade. Although we are interested in financial markets in general (stocks, foreign exchange, etc.), we will use the language of stock markets for the sake of convenience. At each time step the agents make trading decisions $+1,-1$, or 0 , which represent buying or selling a unit of stock, or taking a holding position, respectively. Denoting the decision of agent $i$ at time $t$ by $a_{i}(t)$, the position $k_{i}(t)$ of agent $i$ at time $t$ is given by

$$
k_{i}(t)=\sum_{t^{\prime} \leq t} a_{i}\left(t^{\prime}\right) .
$$

Positive and negative $k_{i}(t)$ refer to a long and short position, respectively.

Introducing the positions of the agents provides a simple way to model the effects of the limited wealth of the agents in real markets. In previous models, the amount of stocks bought (sold) by an agent following each decision is a fraction of her capital (stock) [6,7]. Here, to decorporate the limitations of wealth in the presence of both long and short positions, we define a maximum position $K$ by imposing the constraint, i.e., $\left|k_{i}(t)\right| \leq K$. Once the maximum position is reached, decisions which further increase the magnitude of the positions are ignored. A consequence of this model of trading behavior is that wealth accumulation is additive, in contrast to other models in which wealth accumulation is multiplicative [6,7].

The stock price should evolve according to their demand and supply. Thus, the price of one unit of stock is updated by

$$
P(t+1)=P(t)+\operatorname{sgn}[A(t)]|A(t)|^{\gamma},
$$

where $A(t) \equiv \sum_{i} a_{i}(t)$ represents the excess demand. When there are more (less) buyers than sellers, $A(t)$ is positive (negative) driving the price up (down) according to Eq. (2). The exponent $\gamma$ describes the sensitivity of price increment to the excess demand, $\gamma=0$ and 1 for the cases of step [2-4] and linear functions [9], respectively, which was extensively studied in the literature. On the other hand, there is evidence for $\gamma \approx 0.5$ [16].

In market clearing processes, there is usually a discrepancy between the price expected by an agent when she submitted her bid and the actual transaction price [11]. This discrepancy arises from the sequential process of clearing the deals at each time step, during which the transaction price changes from its present value to the new value according to Eq. (2). For simplicity, we assume that the transaction price is the same for all transactions at time $t$. Thus, we approximate the transaction price $P_{T}$ as

$$
P_{T}(t)=(1-\beta) P(t)+\beta P(t+1),
$$

with $0 \leq \beta \leq 1$. The variable $\beta$ acts as a market impact factor, since it arises from the collective effects of agent participation, and reduces the profit of the majority of the participating agents relative to the so-called price takers. When $\beta=1$, agents experience full market impact in transactions and are trading with the next price. When $\beta=0$, agents experience no market impact in transactions and are trading with the immediate price.

With this evolving transaction price, we let $w_{i}(t)$ be the wealth of agent $i$ at time $t$ just after the decision $a_{i}(t)$ is carried out and the transaction is completed. The wealth $w_{i}(t)$ is the sum of cash $c_{i}(t)$ and stock values in hand, namely,

$$
w_{i}(t)=c_{i}(t)+k_{i}(t) P_{T}(t),
$$

with $k_{i}(t)$ given by Eq. (1). The second term of Eq. (4) corresponds to the stock values in hand, which is the product of the position held and the current transaction price. $P_{T}(t)$ instead of $P(t)$ is employed in calculating wealth, since it represents the actual stock value once decisions are implemented. Cash is updated according to the buying and selling of stocks at time $t$, namely,

$$
c_{i}(t)=c_{i}(t-1)-a_{i}(t) P_{T}(t) .
$$

Suppose agent $i$ buys (sells) a unit of stock at time $t$. Her cash is lowered (raised) by an amount $P_{T}(t)$ while her value of stocks in hand is increased by the same amount, so that any change in wealth is due to the change in value of the 
stocks she previously held. After rearranging Eqs. (2)-(4), the wealth change of agent $i$ after transactions at time $t$ can be expressed as

$$
w_{i}(t)-w_{i}(t-1)=k_{i}(t-1)\left[P_{T}(t)-P_{T}(t-1)\right] .
$$

Next, we consider the strategies used by the agents to reach their decisions in the evolving market environment. In this paper we will consider endogenous environments. In this case, the market environment at time $t$ is described by $\mu(t)$, which is one of the $2^{m}$ strings of the $m$ most recent outcomes of the sign of price changes. Thus, $m$ is called the memory size.

A strategy prescribes the decisions $+1,0,-1$ in response to each of the $2^{m}$ states of the market environment. Each agent draws $s$ strategies randomly at the beginning of the game. Every strategy should have at least one buying and selling decision. At every time step, each agent selects the most successful strategy among the $s$ strategies she owns and uses it to make a decision. The success of a strategy is measured by the virtual wealth it should have acquired were its decisions followed in the market history. This means that for strategy $\sigma$, we start with the initial virtual wealth $w_{\sigma}(0)=0$ and update their values in the same way as we do for real wealth, that is,

$$
\begin{gathered}
k_{\sigma}(t)=\max \left\{-K, \min \left[K, k_{\sigma}(t-1)+a_{\sigma}(t)\right]\right\}, \\
w_{\sigma}(t)=w_{\sigma}(t-1)+k_{\sigma}(t-1)\left[P_{T}-P_{T}(t-1)\right],
\end{gathered}
$$

where $P_{T}$ is the transaction price determined from the real transactions in Eq. (3). In this way, the virtual wealths of the strategies are calculated as if they were the price takers.

A common way to model the incentives of the agents to participate in the market or otherwise is to allow one of their $s$ strategies to be a zero strategy, such as that adopted in the grand-canonical minority game [6-9]. The zero strategy refers to one with a holding decision irrespective of the market environment. Indeed, this feature can be incorporated into our model. However, as we shall see, the basic behavior remains the same. Hence, unless mentioned explicitly, the zero strategy will not be included in our model.

Unlike the original minority game [2], an agent in this model can hold a long or short position while at the same time the virtual positions of the adopted strategy are updated independently. The use of virtual wealths in evaluating strategies is an essential difference with the conventional models of the minority game, which will be discussed in detail in Sec. VI. The ways of wealth accounting in the current market model and the minority game are summarized in Table 1 in Sec. VI. In the present model, the increment of real wealth as well as the virtual wealths of strategies at each step depends on the historical sum of actions according to Eqs. (1) and (6), and Eqs. (7) and (8) respectively, which implicitly embed a longer memory scale in wealth monitoring and strategy selection than the original minority game. This long memory in wealth monitoring introduces a great complexity in the dynamics of system, which brings in a rich behavior reflecting aspects of real markets.

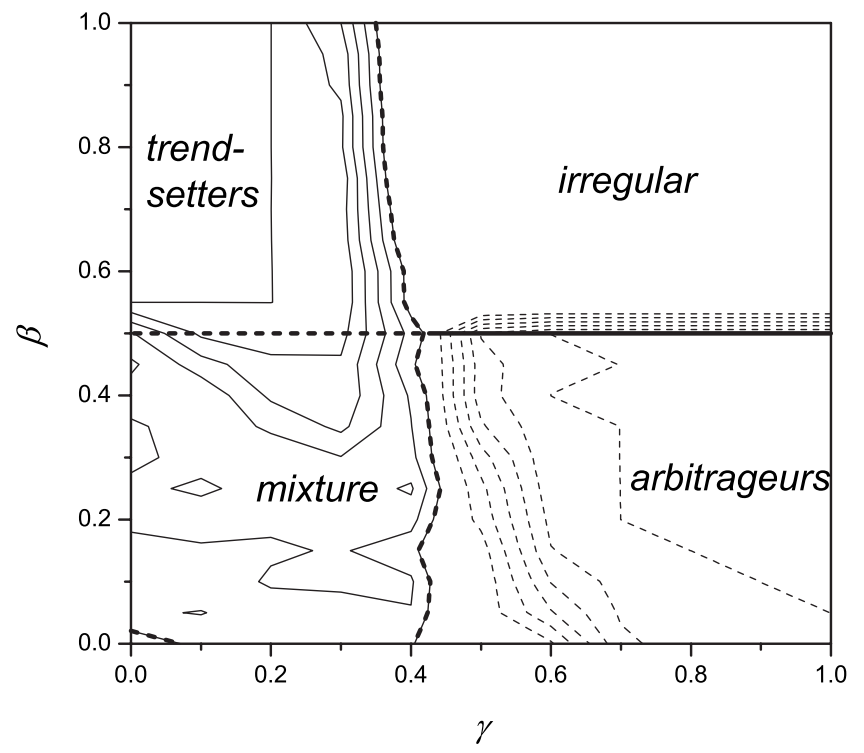

FIG. 1. The phase diagram showing the phases of the trendsetters' attractor, the arbitrageurs' attractor, the mixture phase, and the irregular phase for $m=3, s=2, K=3, N=1000,10^{6}$ steps, and 100 samples. Thick solid (dashed) line: transition discontinuous (continuous) in predictability and volatility. Thin solid (dashed) line: contours of the probability of the trendsetters' (arbitrageurs') attractor.

\section{PHASE DIAGRAM}

In the phase space of price sensitivity $\gamma$ and market impact $\beta$, we found the phases shown in Fig. 1. Examples of the time series of price and the corresponding average wealth are shown, respectively, in Figs. 2 and 3. It can be seen that the system behaves very differently in the arbitrageurs', the trendsetters', and the irregular phases. We will discuss these behaviors in detail in the following subsections.

\section{A. Arbitrageurs' attractor}

In the region with $\beta \leq 0.5$ and sufficiently large $\gamma$, the system eventually falls into periodic attractors with agents buying and selling according to the self-generated price change in a recursive manner. In most cases, the system gets attracted to a period-two cycle in which the same groups of agents synchronize into buying and selling at consecutive time steps, which drives the price oscillating between a higher and lower value accordingly, as shown in Fig. 2(a). Suppose the prices in the period-two cycle are $P_{0}$ and $P_{0}$ $+\left|A_{0}\right| \gamma$. Then the transaction prices become $P_{0}+\beta\left|A_{0}\right|^{\gamma}$ and $P_{0}+(1-\beta)\left|A_{0}\right| \gamma$. With $\beta<0.5$, agents engaged in this cycle gain $(1-2 \beta)\left|A_{0}\right|^{\gamma}$ per cycle by buying at a lower price and selling immediately at a higher price. These agents can be called the arbitrageurs. The attractor is robust since the net gain in wealth of the arbitrageurs reinforces their use of the so-called smart strategies when the market dynamics is dominated by the buy-sell cycles [17]. The anti-arbitrageurs, who sell at a lower price and buy immediately at a higher price, lose $(1-2 \beta)\left|A_{0}\right|^{\gamma}$ per cycle. Since the virtual wealths of their strategies are much lower, they are outnumbered by 

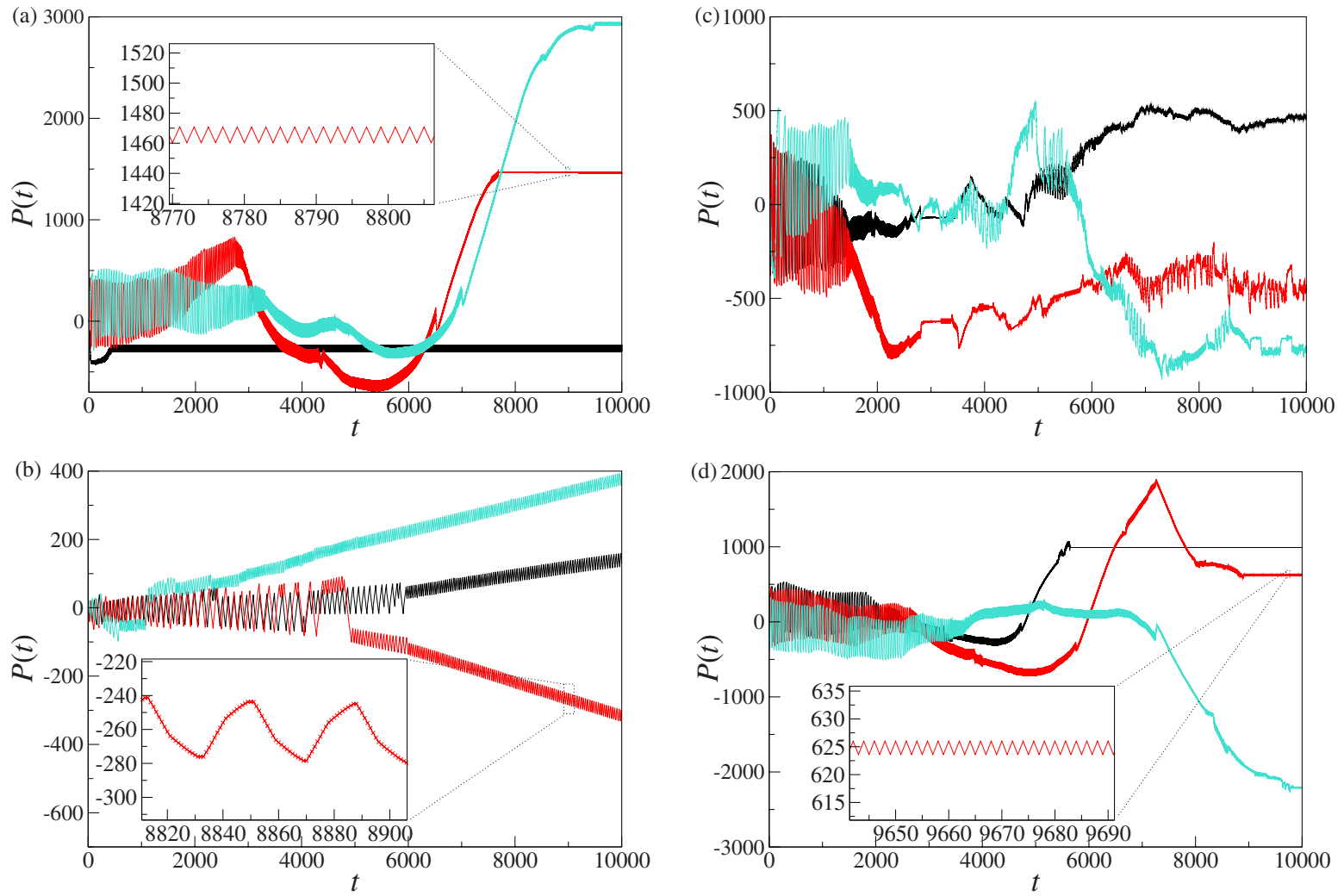

FIG. 2. (Color online) The time dependence of price for three samples with $m=3, s=2, K=1, N=1000$ for (a) the arbitrageurs' phase at $(\gamma, \beta)=(0.8,0.4)$, (b) the trendsetters' phase at $(\gamma, \beta)=(0.2,0.8)$, (c) the irregular phase at $(\gamma, \beta)=(0.8,0.8)$, and (d) the phase boundary at $(\gamma, \beta)=(0.8,0.5)$.

the arbitrageurs. Other agents adopt strategies which have an unequal number of buy and sell decisions in a period-two cycle, and their maximum or minimum positions prevent them from participating in the market.

The behaviors of the arbitrageurs are reminiscent of those of the privileged traders in real financial markets. They place their buying (selling) bids in the market, stimulating price rises (drops). The regime of low market impact corresponds to the dominance of these traders, who have the privilege to have their bids cleared earlier than the other traders, and hence enjoy a more favorable price before the price moves significantly. At the next step, they place an opposite bid in the market, stimulating a price change in the reverse direction, and recovering their cash (stocks) with a profit margin.

As shown in Fig. 3(a), the average wealth of the agents increases linearly with time. When $\beta=0.5$, the agents buy and sell at the same price in the cycle, resulting in zero change in their average wealth after the transients have subsided, as shown in Fig. 3(d).

A phase transition takes place at $\beta=0.5$, since in the regime of large market impact with $\beta>0.5$, agents engaged in similar buy and sell cycles are eventually losing as they are buying at a higher price and selling at a lower price. As shown in Fig. 4(a), the average wealth gain of the agents changes from positive to negative at $\beta=0.5$ for $\gamma=0.5$ and $K=1$. The change is accompanied by a change in the slope, indicating that a continuous phase transition is taking place, and can be attributed to the disappearance of the arbitrageurs' attractor at $\beta=0.5$.
Furthermore, we monitor the predictability $H[18]$ and the volatility $\sigma[3]$. The former is defined as

$$
H=\sum_{\mu} \rho(\mu)\langle\delta P \mid \mu\rangle^{2}
$$

where $\langle\delta P \mid \mu\rangle$ is the average of the price change conditional on state $\mu$, and $\rho(\mu)$ is the probability of occurrence of state $\mu$. The volatility $\sigma$ of price changes is defined as
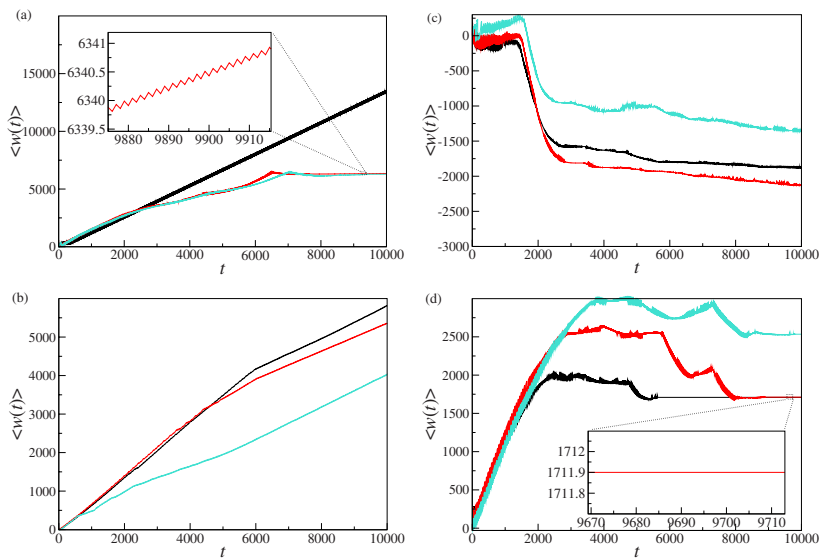

FIG. 3. (Color online) The time dependence of the average wealth for the corresponding samples in Fig. 2. 

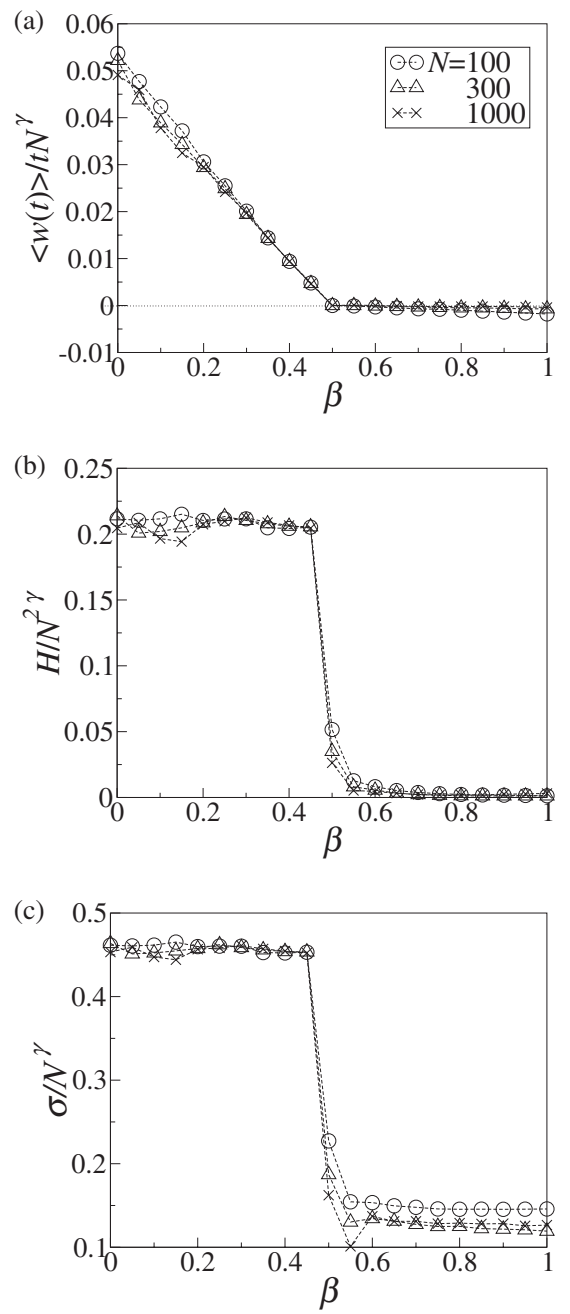

FIG. 4. The dependence on $\beta$ of (a) the average wealth gain per step, (b) the predictability, and (c) the volatility, for $\gamma=0.5$ and $K$ $=1$. Simulations are performed with $N=100,300,1000,10^{5}$ steps, and 1000 samples.

$$
\sigma=\sqrt{\left\langle\delta P^{2}\right\rangle-\langle\delta P\rangle^{2}}
$$

Since the dynamics of the arbitrageurs' attractor is embedded in a low dimensional space, we expect that the excess demand $A$ scales as $N$ in this regime [19,20]. Consequently, we expect that $H$ and $\sigma$ scales as $N^{2 \gamma}$ and $N^{\gamma}$, respectively. As shown in Figs. 4(b) and 4(c), the predictability vanishes and the volatility is greatly reduced for $\beta$ above 0.5 , supporting the pictures of a phase transition.

\section{B. Trendsetters' attractor}

\section{Dynamics}

The phase space is dominated by the trendsetters' attractor for sufficiently low $\gamma$. It is quasiperiodic, an example being shown in Fig. 5. A trendsetter strategy of memory size $m$ consists of a buying decision responding to a signal with $m$ consecutive instants of rising price, and a selling decision responding to a signal with $m$ consecutive instants of dropping price. The trendsetters' attractor phase is increasingly prominent when the maximum position $K$ increases. For a large $K$, the agents are allowed to hold multiple short or long positions in hand. This increased freedom allows them to gain by holding a long (short) position when the price is rising (dropping). As shown in Fig. 5(a), this strategy enables one to gain at all time steps in a market dominated by the trendsetters' attractor, except near the points where a rising trend switches to a dropping one, or vice versa. Consequently, the trendsetter strategy is the most successful strategy in a trendsetters' attractor, and the agents holding at least one trendsetter strategy will make decisions accordingly. These agents are referred to as the trendsetters.

The dynamics of the attractor consists of four stages:

(1) In the first stage, the trendsetters make buying decisions collectively. Starting from short positions, they accumulate stocks step by step and switch from minimum to maximum positions in $2 K$ steps. At the end of this stage, the
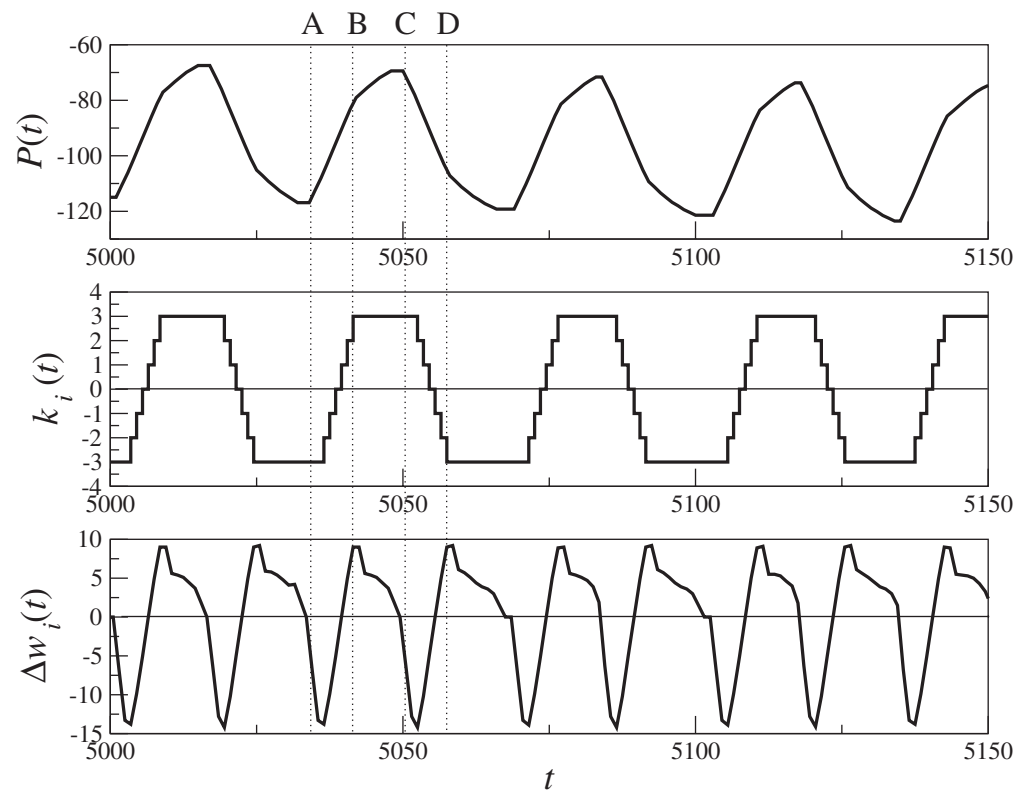

FIG. 5. An example of the trendsetters' attractor showing (a) the price, (b) the position of a typical trendsetter, and (c) the wealth of a typical trendsetter. The first to fourth stages start at points $A, B, C, D$ respectively. Parameters are $m=3, s=2, K=3, \gamma=0.3, \beta=0.8$, and $N=1000$. 
positions of the trendsetters reach the maximum, and they are restrained from further buying actions.

(2) In the second stage, another group of agents takes up the role of collective buying and pushes the price further up. This group consists of the fickle agents, who fickle their decisions between two strategies. Through the adaptive process of trading, the virtual wealths of the two strategies adjust to such levels that the selling decisions of a strategy are favored in the first stage, and the buying decisions of another strategy are adopted in the second stage. This causes the fickle agents to take buying actions in the second stage, pushing the price further up. At the end of this stage, the positions of the fickle agents reach the maximum, and they are restrained from further buying actions. The market will then remain quiet with no price movement, waiting for a random signal to drive the next stage.

(3) The third stage is triggered by a randomly generated selling signal, and the trendsetters respond by collective selling decisions. Starting from long positions, they cash in stocks step by step and switch from maximum to minimum positions in $2 K$ steps. At the end of this stage, the positions of the trendsetters reach the minimum, and they are restrained from further selling actions.

(4) In the fourth stage, the fickle agents switch their strategies to selling, since the falling price in the third stage reduces the virtual wealth of the buying strategies and boosts that of the selling strategies. At the end of this stage, the positions of the fickle agents reach the minimum, and they are restrained from further selling actions. The market will then remain quiet with no price movement, waiting for a random signal to drive another cycle.

\section{Stability}

The stability of the attractor depends on whether the trendsetters are able to increase their wealth in a cycle. Consider the case of $m=2$, for example. The fast trendsetters respond to the signals of drop-rise and rise-drop with buying and selling decisions, respectively. They detect the change in the trend at the earliest possible instant and become the first group of trendsetters to benefit in the first and third stages.

On the other hand, the slowest trendsetters respond to the signals of drop-rise and rise-drop with selling and buying decisions, respectively. They only join the other trendsetters $m-1$ steps after the onset of a trend. Hence, they lose wealth due to their failure to respond timely to the changing trend in the first and third stages.

However, since the fickle agents push the price further up and down in the second and fourth stages, respectively, the slowest trendsetters have the opportunity to regain their wealth through holding long and short positions, respectively. So in other words, if the price change caused by the fickle agents is sufficient to ensure the slowest trendsetters to gain wealth in a cycle, the trendsetters' attractor will become stable.

The stability is achievable when the price sensitivity $\gamma$ is sufficiently low. Low values of $\gamma$ imply that the return in a step is rather insensitive to the excess demand. Since the virtual wealth of the strategies held by the fickle agents is lower than that of the trendsetters, the population size of the fickle agents is less than that of the trendsetters. Hence, if the return is insensitive to the excess demand, the price change caused by the fickle agents in the second and fourth stages will not be too much less than those caused by the trendsetters in the first and third stages. The slowest trendsetters will then be able to regain their wealth. Consequently, the trendsetter's attractor is only stable for sufficiently low values of $\gamma$.

While the analysis for the general case is not available so far, we have considered the simple case of $m=2$, which captures the essentials of the agent dynamics. In this case, the number of kinds of agents is few enough for a systematic analysis. By carefully tracing the decisions of the different types of agents in this essential model, one can deduce the price change and the wealth gained by the different types of agents in the attractor, and hence the stability condition of the trendsetters' attractor. Since the analysis is lengthy, details will not be presented here. Nevertheless, we mention that the resultant condition, in the form $\beta \leq \beta_{\text {trendsetter }}(\gamma)$, has a remarkable agreement with the simulation results.

It is interesting to consider the stability of the trendsetter attractor in the grand-canonical version of our model market, since it depends on the fickle agents who continue to lose wealth to the trendsetters. Consider the grand-canonical market in which each agent has an extra zero strategy, which receives a payoff of $\epsilon$ at every time step and whose decisions are zero irrespective of the environment; $\epsilon$ is the interest rate. This allows the agents to refrain from playing, if their other strategies do not grant them a positive return. Since the fickle agents in the trendsetter attractor lose wealth, one might expect that they will withdraw from the market, destabilizing the attractor.

Surprisingly, we find that the trendsetter attractor continues to exist in the grand-canonical market. We monitor the strategies of the fickle agents, and find that their strategies are in fact winning strategies averaged over time. Hence, the fickle agents do not use the zero strategy. However, they switch between the good strategies in an untimely manner, causing them to lose wealth even though their strategies are gaining when averaged over time.

Furthermore, we note that the market behavior is rather sensitive to the interest rate in the original version of the minority game. For example, the predictability drops significantly when the interest rate changes from 0 to a small positive value [21]. In our model, the market behavior in the grand-canonical setting is effectively the same as that in the canonical one when the interest rate is around 0 , as indicated by the wealth gain per step, predictability, and volatility. Even at an interest rate as high as 0.1 , the trendsetter attractors continue to exist, although the wealth gain per step, predictability, and volatility of the market are gradually reduced in the negative-sum regime. The insensitivity to the interest rate in our model is probably due to the use of wealth-based payoff schemes, and the trendsetters' phase is in the regime of positive sum.

\section{Dependence on $\gamma$}

Figure 6 shows the average wealth gain per step, the predictability, and the volatility when $\gamma$ increases across the 

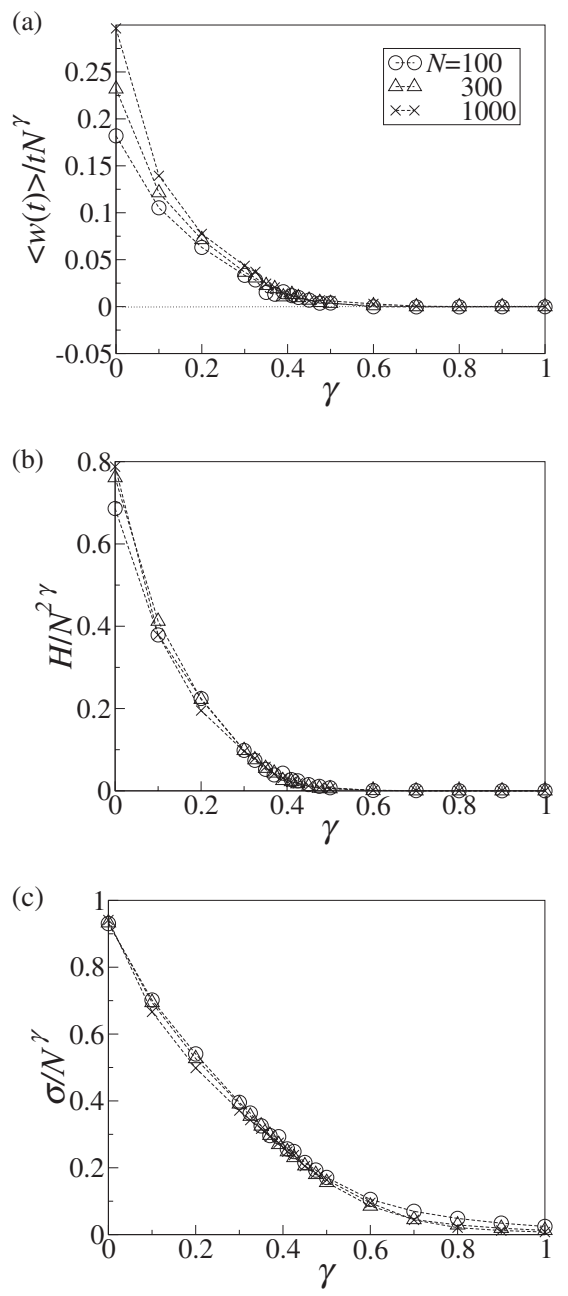

FIG. 6. The dependence on $\gamma$ of (a) the average wealth gain per step, (b) the predictability, and (c) the volatility, for $\beta=0.8$ and $K$ $=3$. Simulations are performed with $N=100,300,1000,10^{6}$ steps, and 100 samples.

phase boundary separating the phases of the trendsetters' and irregular attractors. There appears a change in the slope of the wealth gain per step in Fig. 6(a) when the phase boundary is crossed, but no discontinuities seem to appear in the predictability and volatility in Figs. 6(b) and 6(c). This indicates that the phase transition of the trendsetters' attractor is milder than that of the arbitrageurs' attractors.

Another interesting feature of the trendsetters' attractor is that the price does not return to the same value after one period. This contributes to a constant growth or decay rate after averaging over many periods. We have also studied the sample-averaged growth rate of the price, and found that it also scales as $N^{\gamma}$. After rescaling by $N^{\gamma}$, the dependence of the growth rate of the price is qualitatively similar to those in Figs. 6(a)-6(c).

In the region where both the trendsetters' and the arbitrageurs' attractors are stable, we have a mixture phase. The type of attractor reached in the steady state depends on the sample. In this mixture phase, some trendsetters' attractors are quasistable in the transients, and eventually settle as arbitrageurs' attractors in the steady state.

\section{Relation to other models}

The behavior of the trendsetters' attractor is very similar to the bubbles and crashes found in [6]. There, the trendsetters first create a rising trend, which subsequently flattens because of their limited buying power. Then, the so-called fundamentalists act contrarily to the main trend, triggering the crash. It is inspiring to note the universality of behavior in these two models, despite the different ways of introducing the relevant parameters. In our model, the trendsetters' attractor exists only when the price sensitivity $\gamma$ is sufficiently low, so that the trendsetters and their followers are able to gain wealth at the end of the rising (dropping) trend by having long (short) positions. On the other hand, the crashes and bubbles in [6] are sustainable only when the fraction of investment per agent per step is sufficiently small. We note that both the price sensitivity in our model and the investment fraction in [6] play the role of transducing the excess demand to price movement.

Moreover, both models contain mechanisms for trend reversal. In [6], trend reversals are triggered by the fundamentalists, whereas in our model trend reversals at the end of rising or dropping trends become unavoidable since the positions of the agents have reached their maximum or minimum.

On the other hand, our model differs from that of [6] in having fewer parameters, having the market makers, and having no reference return. The robustness of the trendsetters' attractor despite these differences shows that it is a ubiquitous phenomenon in certain parameter ranges. As a further step, we have described the role played by the different types of agents in the self-organization of these attractors.

The behaviors of the agents in the trendsetters' attractor resemble those in real financial markets. The trendsetters respond to a bullish signal and take buying actions collectively, creating a bullish market. They stop buying due to their finite capital or assessment of risks, followed by the fickle agents who try to catch up. Eventually the market becomes quiet when all agents have exhausted their capital or tolerance to risks. Then a bearish signal appears and the trendsetters sell their stocks, followed by the fickle agents.

\section{Irregular phase}

In the phase diagram of Fig. 1, there is an irregular phase in which the periodic and quasiperiodic attractors cannot persist. The trendsetters' attractor appears only intermittently, since they are not stable in the long run. Other intermittent states are observed. Abrupt and sudden changes are found connecting the intermittent states.

For $\gamma<1$, long term price fluctuations embody fluctuations in shorter time scales. An example is shown in Fig. 7(a). The trendsetters' attractors appear in some transient and intermittent states, especially in the early stage, but not persistently. The bottom inset shows the time interval in which the system does not lie in the trendsetters' or the arbitrageurs' attractors, showing the fluctuations in short time scales embodied in longer time scale fluctuations.

The case of $\gamma=1$ is special. Since the price change is linearly related to the global sum of actions $A(t)$, the price 

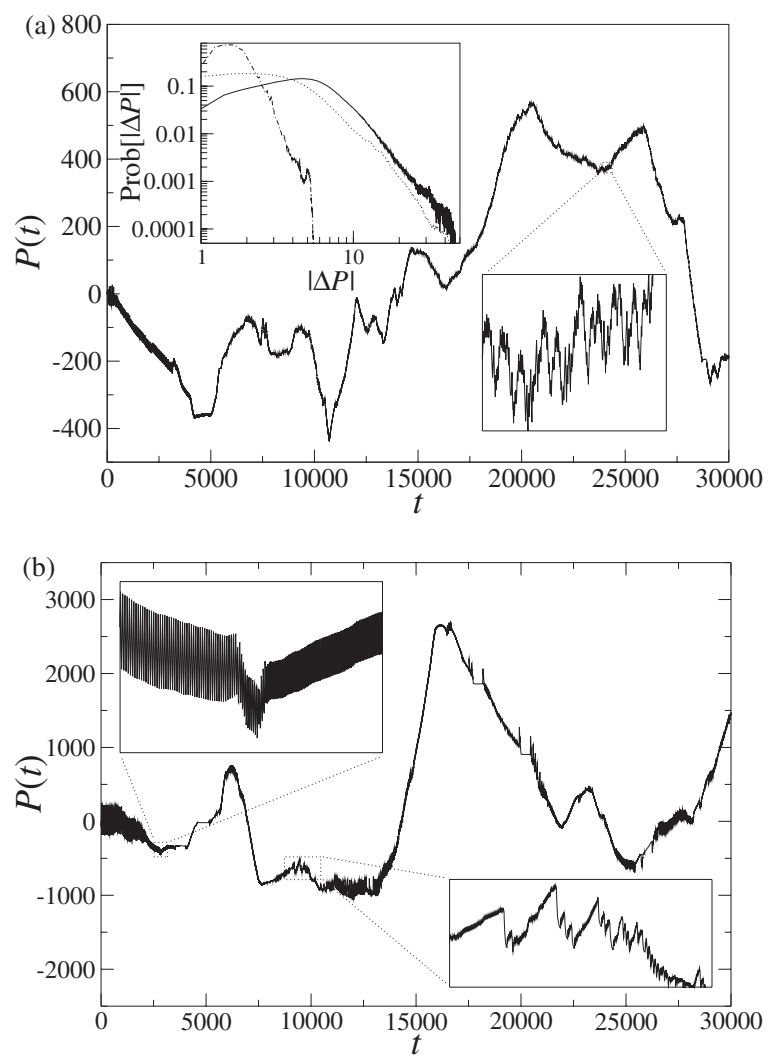

FIG. 7. (a) An example of the time dependence of the price at $\beta=0.8, m=3, s=2, \gamma=0.7, K=1$, and $N=1000$. Inset: The volatility distribution of price with $N=1000, \beta=0.8$ and $m=3,(K, \gamma)$ $=(1,0.3)$ (dash-dotted line), and $(K, \gamma)=(3,0.7)$ (dotted line). Solid line: $N=10000, m=6, s=2, \gamma=0.5$, and $K=3$. (b) Another example of the time dependence of price at $K=3$, other parameters the same as in (a).

trend in the long run is flat, consequential to the limit $\lim _{t^{\prime} \rightarrow \text { inf }} \Sigma_{t^{\prime}}^{t} A\left(t^{\prime}\right) \approx 0$ for finite maximum positions. Thus, the condition $\gamma<1$ is essential in reproducing the nonstationary price trend commonly found in real financial markets.

The inset of Fig. 7(a) shows the corresponding distribution of price change, with the fat tail of the distribution obeying a power law. For the leftmost curve, the exponent is approximately -7.4 , resembling the fat tail volatility distribution in real financial markets [22]. The sharp drop at the end of the tail is due to finite size limitations in simulations. The other curve in the same figure shows that increasing $\gamma$ causes the distribution of fluctuations to spread out, and the fat tail decays more gently.

Figure 7(b) shows another example of the time series of price in the irregular phase with $K=3$. As shown in the top inset of Fig. 7(b), the system switches from one quasiperiodic cycle to another, with an intermediate state in-between. The trendsetters' attractors appear in the transients, but not persistently. The bottom inset of Fig. 7(b) shows that fluctuations are present in multiple time scales. The price volatility distribution in this region confirms a fat tail distribution obeying a power law of exponent -3.8 , as shown by the solid line in the inset of Fig. 7(a).

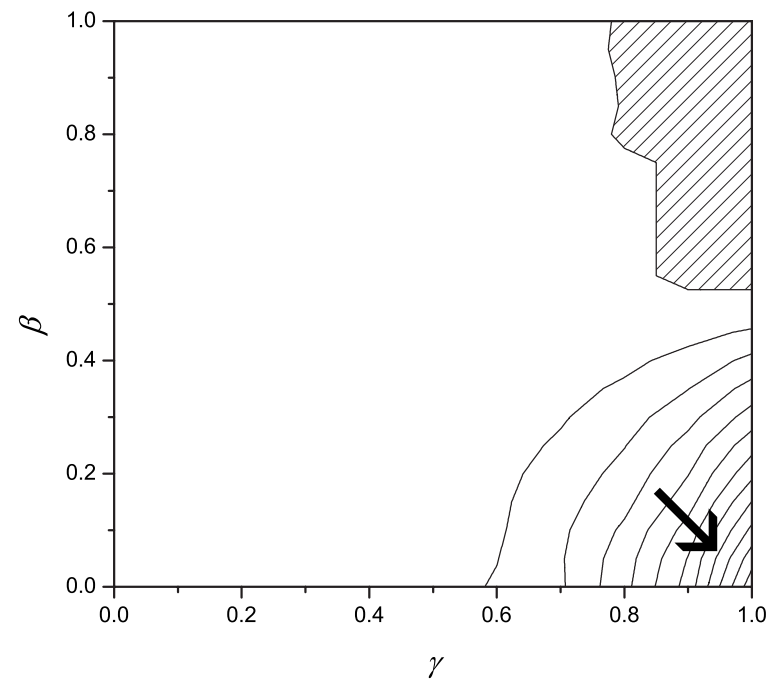

FIG. 8. The average wealth gain per step of the agents after equilibration as a function of $\gamma$ and $\beta$ for $m=3, s=2, K=3, N$ $=1000,10^{6}$ steps with 400 samples. Each contour represents an increment of 2. The shaded region corresponds to the region of negative average wealth. The direction of the arrow indicates the direction of increasing wealth gain.

\section{Positive and negative sumness}

The average wealth of the agents in the space of $\gamma$ and $\beta$ is shown in Fig. 8. For $K=3$, the region of positive average wealth dominates the space. This means that the market is a positive-sum game for the agents. Much of the positive sum region is covered by the region of the two attractors, as can be seen from a comparison with Fig. 1. Besides that, there exist regions of positive sums in the irregular phase. The behavior of this region resembles the real financial market: agents have a positive gain on average, while the dynamics is not periodic. Furthermore, the volatility distribution of the price follows a fat tail distribution with a power law exponent -4 in the case of $K=3$, not very far from those observed in real financial markets.

This positive sumness adds a new perspective to conventional market models, such as the original minority game, which has a negative sum in terms of the number of winners. We remark that the positive sumness is a natural consequence of the model where artificial means, such as capital injection, are not employed to ensure profitability. In real markets, this is essential to resolving the issue of attracting agents to participate, were they given the option.

As shown in Fig. 8, the average wealth decreases when $\beta$ increases, since the market impact reduces the profit of the transactions. Furthermore, the effect of the market impact on price movements is hardly felt if the price sensitivity $\gamma$ is not sufficiently high. Hence the region of negative sumness is only found at high values of both $\beta$ and $\gamma$. This feature also depends on the maximum position $K$. In general, the region of negative sum shrinks with $K$, as shown in Fig. 9.

\section{E. Role of the market makers}

To understand the origin of the positive sumness, we note that supply and demand in the market is not balanced. When 


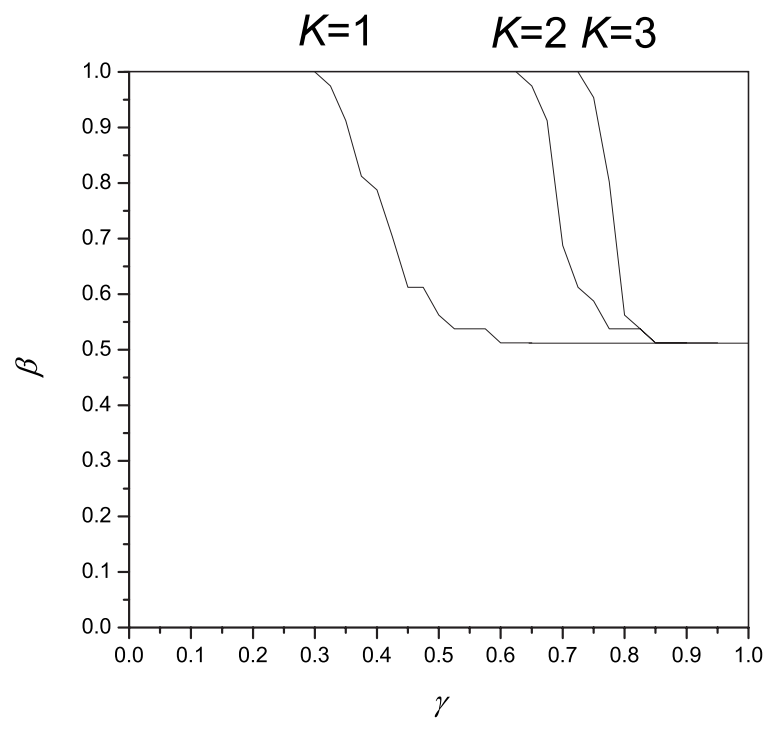

FIG. 9. The regions of positive and negative sumness in the space of $\gamma$ and $\beta$ for $m=3, s=2$. Simulations are performed with $N=1000,10^{5}$ steps with 400 samples.

there are more buyers than sellers, we implicitly assume someone is willing to provide the extra stocks for the buyers. Likewise, when there are more sellers than buyers, we implicitly assume someone is willing to absorb the extra stocks from the sellers. This role of clearing the extra supply and demand at every time step is played by the market makers. At every time step, the market makers take actions opposite to the collective actions of the agents. It results in an exactly zero-sum game if we sum up the wealth increments of all the agents and the market makers. Although the overall wealth is zero, the phase diagram in Fig. 1 actually shows the wealth shifted from the market maker to the players, which contribute to the positive sumness of the players. It serves as a main allure for individual players to participate in the market. However, the positive sumness of the players implies the negative sumness of the market makers, and the issue of voluntary participation shifts to why the market makers have incentives to participate in the market. Hence, there should be additional mechanisms bringing positive gain to both players and the market makers. Before we address this issue in the next section, we consider the case of markets without the market makers.

\section{F. Markets without market makers}

To model markets without market makers, we balance the supply and demand at every time step by randomly drawing agents from the majority side to match the minority side, whereas the excess demand before matching is still used in the determination of price change. Those who are not fortunate enough to carry out their decisions are forced to reset their actions to zero for the time being. As a result, the number of actual buyers and sellers will be equal at every step. Among the agents, the total number of long positions is always equal to the total number of short positions, leading to a zero-sum game among the agents.
The price trend in this model is very monotonic. This can be attributed to the extra buyers (sellers) whose decisions are rejected by the market. While their decisions are frustrated, their bids nevertheless drive the price up (down), leading to a signal of rising (dropping) price at the next step. With their frustrated decisions, they repeat their decisions and buy (sell) again. The signal of rising (dropping) price continues, and eventually all agents making the opposite decision are exhausted. Driven by this extra demand or supply, the price goes either up or down continuously, but there are no transactions. This behavior resembles the "stubborn majority" in the \$-game [5] except that there are no market makers to be arbitraged in the present case. The scenario is familiar in the real estate market of Tokyo in the early nineties or Hong Kong in the late nineties, in which real estate prices are driven by wild speculations to an unaffordable level. Alternatively, a continuously dropping market with no transactions is similar to the real estate market after the burst of the bubble.

While our model is successful in reproducing these realistic features, it has not included mechanisms that terminate the indefinitely rising or dropping trends. This constitutes a separate issue beyond the scope of this paper.

\section{PROFIT OF THE MARKET MAKERS}

In the real market, the market makers should have a positive gain in return for balancing the supply and demand. This positive gain encourages them to maintain their service in the market. It was proposed that the market makers may lower the risk of being arbitraged by the players through adding their inventory to the excess demand in the determination of price [5]. However, we found that this method cannot generate profit for the market maker in the present model. Hence, we consider the alternative proposal that the market makers impose a bid-ask spread during their transactions with the players. That is, the transaction prices at time $t$ are $P_{T}(t) \pm S(t)$ for the buying and selling agents, respectively, where $2 S(t)$ is the bid-ask spread at time $t$. Obviously, imposing the spread makes it possible for the market maker to gain a profit from the transactions. However, to ensure that both the agents and the market makers have incentives to participate, the bid-ask spread must be determined in such a way that the wealth of both the agents and the market makers are stable in time. Here, we consider three ways of determining the spread and compare their effectiveness with respect to this objective.

(1) Fixed bid-ask spread-Figure 10(a) shows the average wealth per agent for different fixed values of $S(t)=S$. Since the total wealth of the agents and the market makers sum up to zero, a positive wealth of the agents implies a negative wealth of the market makers, and vice versa. We can see that the wealth of the agents decreases with the spread. When the spread is sufficiently large, the wealth of the agents becomes negative at the end of the monitoring period, implying that the market makers are getting a profit. However, in the framework of fixed transaction costs, there is only one optimal transaction cost which yields a zero rate of wealth gain of the agents asymptotically [such as that around 0.4 in Fig. 

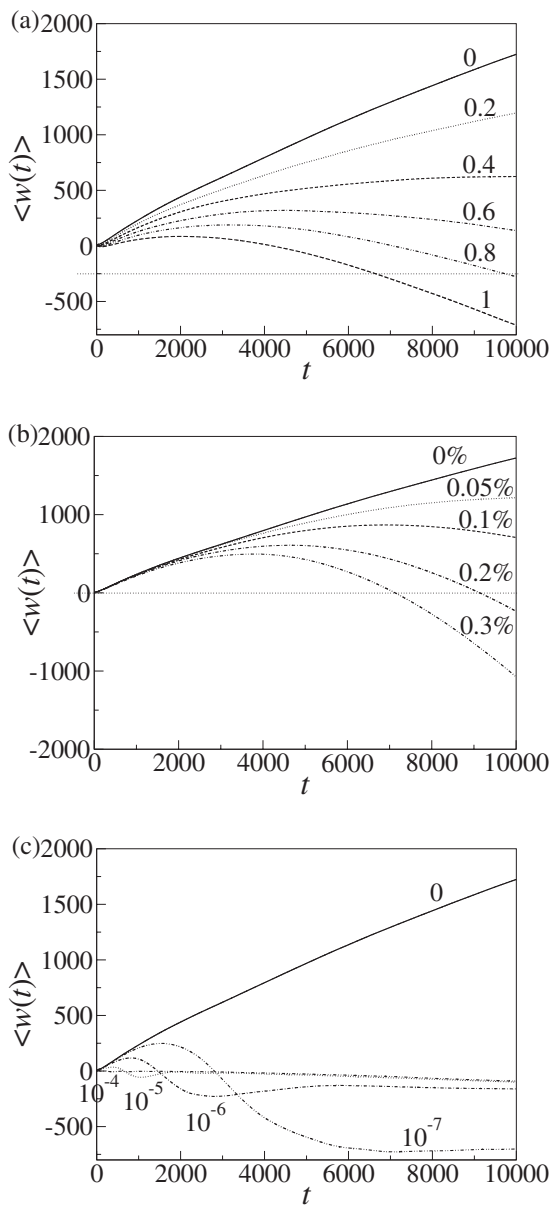

FIG. 10. The time dependence of the average wealth of the agents for $m=3, s=2, \gamma=0.5, \beta=0.5, K=1, N=100$, and 1000 samples for (a) fixed bid-ask spread at the indicated values of $S$, (b) fixed transaction rate at the indicated values of $R$, and (c) adaptive transaction rate at the indicated values of $\eta$.

10(a)], and it is difficult for the market to know this optimal cost a priori. Even for this optimal cost, the approach to the asymptotic is too slow. Consequently, a fixed bid-ask spread cannot ensure a stable balance of wealth between the agents and the market makers.

(2) Fixed transaction rate-For the market makers to maintain a profit in the long run, we consider a fixed transaction rate $R$ given by $S(t)=R\left|P_{T}\right|$. As shown in Fig. 10(b), the rate of change of the average wealth of the agents decreases with time. This is due to the increasing magnitude of the price with time, so that the spread collected by the market makers increases with time. Hence, for a sufficiently high transaction rate [such as $0.3 \%$ in Fig. 10(b)], the market makers gain a profit at the end of the monitoring period shown in Fig. 10(b). Even for a modest transaction rate [such as $0.1 \%$ in Fig. 10(b)], it is anticipated that the agents will eventually lose to the market makers. For a low transaction rate [such as $0.05 \%$ in Fig. 10(b)], we are not certain whether the average wealth of the agents will continue to increase, eventually saturate, or finally decrease beyond the monitoring period. In any case, the time it takes to reach a steady balance of wealth between the agents and the market makers,

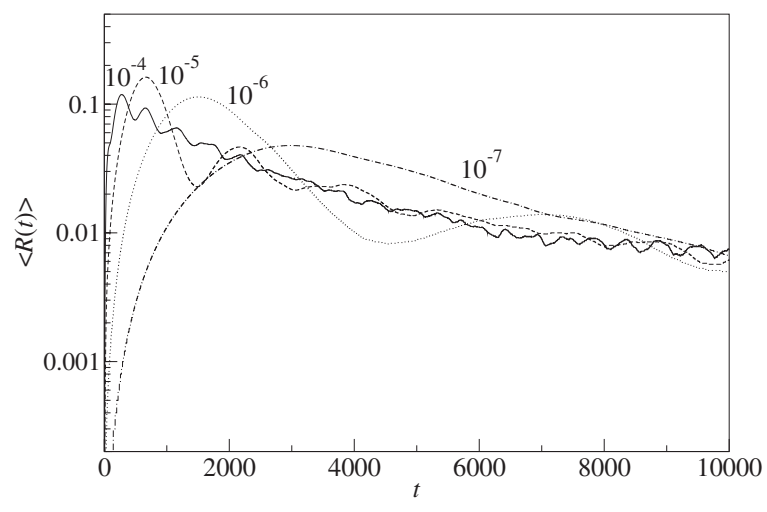

FIG. 11. The time dependence of the sample-averaged transaction rate for $\eta=10^{-7}, 10^{-6}, 10^{-5}$, and $10^{-4}$. Parameters are the same as those in Fig. 10.

if it exists at all, is apparently prohibitively long.

(3) Adaptive transaction rate-Since the above two methods cannot ensure a stable balance of wealth between the agents and the market makers, we introduce an adaptive transaction rate as follows. For a bid-ask spread given by $S(t)=R(t)\left|P_{T}(t)\right|$, suppose the market makers decide the transaction rate $R(t)$ so as to attain a total target wealth of $W_{\text {target }}(t)$ at time $t$. Since their total wealth at time $t$ is $-\sum_{i} w_{i}(t)$, they would increase (decrease) $R(t)$ if $W_{\text {target }}(t)$ is greater (less) than $-\sum_{i} w_{i}(t)$. Hence, we propose an adaptive transaction rate updated according to

$$
R(t+1)=\max \left\{R(t)+\frac{\eta}{N}\left[W_{\text {target }}+\sum_{i} w_{i}(t)\right], 0\right\},
$$

where $\eta$ is the learning rate, dependent on how soon the market makers would like to reach their target wealth. Here, we consider the case that the target wealth approaches zero. This is the scenario in which there is a fierce competition among the market makers. Results in Fig. 10(c) show that the average wealth of the agents (and hence that of the market makers) converges to a steady value after the transients die out, the duration of the transient period decreasing with the learning rate. Figure 11 shows the sample-averaged transaction rate, indicating that it is necessary to impose a high transaction rate in the transient period, before $R(t)$ approaches a universal trend effectively independent of the learning rate. This confirms the previous result that it is not possible to maintain a stable balance of wealth between the agents and the market makers using a fixed transaction rate.

\section{NEW PLAYERS IN AN EVOLVING MARKET}

So far, we have found the existence of positive sumness in the space of price sensitivity and market impact, and that an adaptive transaction rate can ensure a stable balance of wealth between the agents and the market makers. However, the model is still a zero-sum game, and one cannot keep both the agents and the market makers happy at the same time. To ensure participation incentives of both groups, we consider in this section open markets, in which underperforming 

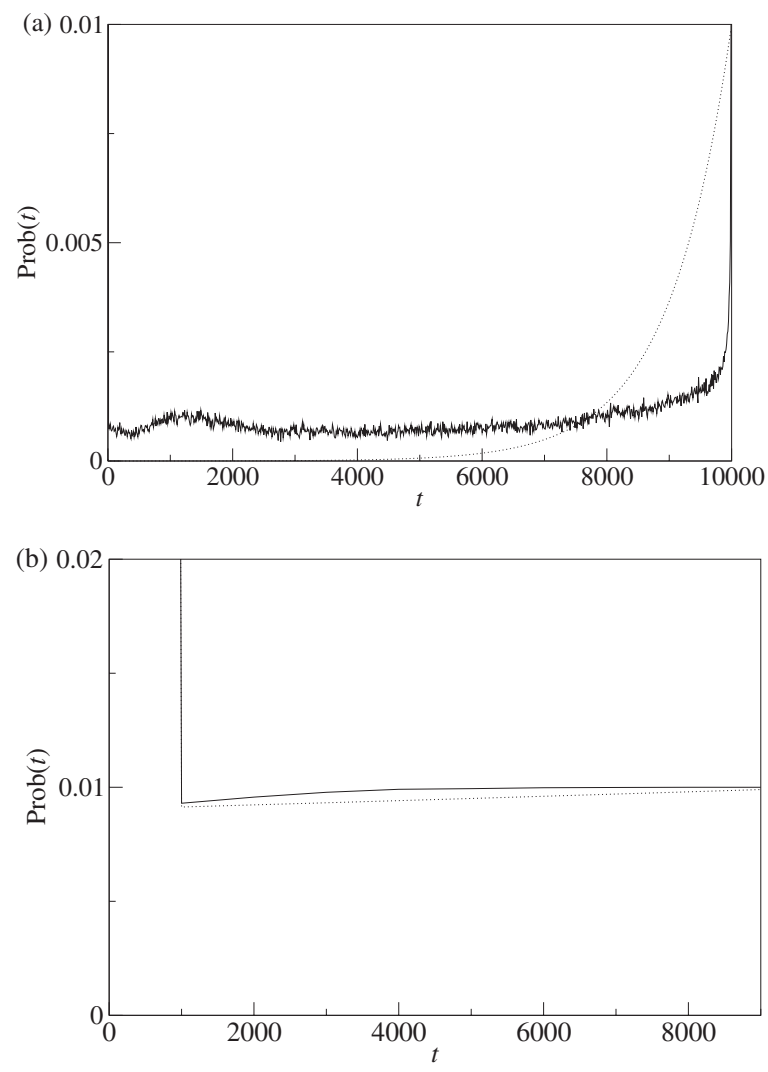

FIG. 12. The survival probability at time $T=10000$ of new players entering the market at time $t$ for $m=3, s=2, K=3, \gamma=0.5, \beta$ $=0.5, W_{\text {target }}(t)=0, \eta=10^{-5}, N=100$, and 1000 samples, $T_{\mathrm{ev}}=$ (a) 10 , (b) 1000. Dotted line: The corresponding survival probability for random evolution.

agents leave the market. They are replaced by new players who bring their wealth into the market. Hence, for every replacement of an agent, the average growth of wealth of the entire market is equal to the difference between the average initial wealth of a new player, minus the average final wealth of an exiting agent.

One worry concerning the issue of participation incentives is that when the new players enter the market, the old players have already adapted their strategies to trade in the market, and the new players have disadvantages in their effort to profit from the market. If the new players merely play the role of supplying wealth to the market, without themselves any hope to profit from their participation, then the new players will not have incentives to participate in the long run.

Here, we consider the following model of an evolving market. At $t=0, N$ agents start their trading activities with their initial wealth set to zero. At every $T_{\mathrm{ev}}$ time step, the agent with the least wealth will leave the market. She is replaced by a new agent with zero initial wealth and a new set of randomly chosen strategies.

To consider the extent to which the survival probability of the new players is enhanced, we monitor the survival probability at time $T$ of the agents entering the market at time $t$, and compare it with the survival probability of random evolution, which is given by $p_{\text {ran }}(0)=(1-1 / N)^{T / T_{\mathrm{ev}}}$ and $p_{\mathrm{ran}}(t)$

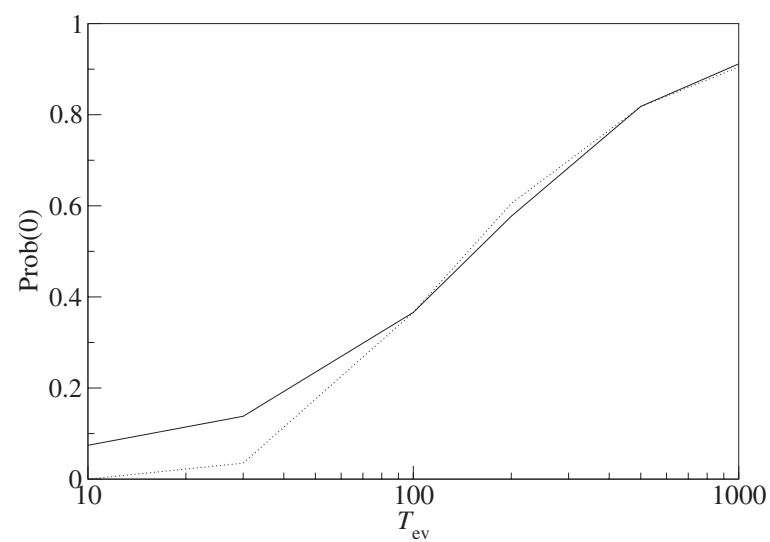

FIG. 13. Solid line: survival probability at time $T=10000$ of the old players as a function of evolution time $T_{\mathrm{ev}}$. Dotted line: The corresponding survival probability for random evolution. Parameters are the same as those in Fig. 12.

$=(1-1 / N)^{(T-t) / T_{\mathrm{ev}}} / N$ for $t>0$. In Fig. 12(a), we observe that the agents entering the market at an early stage of the game have a higher survival probability than random evolution. However, for fast evolution (low values of $T_{\mathrm{ev}}$ ), the most recently entering agents have a much lower survival probability than random evolution. Furthermore, Fig. 13 shows that the survival probability of the old players is higher than that of random evolution for low values of $T_{\mathrm{ev}}$. This shows that the enhanced survival probability of the early agents is not the consequence of gaining wealth from the old players, but rather taking advantage of the recent entering agents. This implies that the late corners will have little incentive to participate in a fast evolving market.

On the other hand, for a slowly evolving market, as shown in Fig. 12(b), we see that the survival probability of the new agents is higher than that of random evolution for most of the entering time of the new agents. This shows that the new agents have learned to play as well as, or even better than, the old players.

Figure 13 shows the survival probability of the old players as a function $T_{\mathrm{ev}}$. The survival probability is higher than that of random evolution for low values of $T_{\text {ev }}$, but is roughly the same for high values of $T_{\mathrm{ev}}$. This again shows that slow evolution provides a fairer environment for the survival of the new players.

We have also tested the case that the new agents enter the market with initial wealth equal to the average wealth of the agents at the moment of entry. This places the new agents on a much more equal footing with the old players. Consequently, the survival probability of the new agents becomes effectively the same as that for random evolution, even at the parameters of Fig. 12(a). This provides another support to the assertion that it is possible for the new agents to have participation incentives.

\section{TESTING WITH HANG SENG INDEX}

To test whether the wealth-based payoff scheme introduced by us is applicable to real market data, we replace the 
TABLE I. The payoff change of strategy $\sigma$ after transactions at time $t$ for the wealth game, the minority game, the \$-game, and the majority game. Here $a_{\sigma}(t)$ is the decision made by strategy $\sigma$ at time $t$.

\begin{tabular}{|c|c|}
\hline & $\begin{array}{l}\text { Wealth change of strategy } \sigma \\
\text { after transactions at time } t\end{array}$ \\
\hline Wealth game & $\begin{array}{l}k_{\sigma}(t-1)\left[P_{T}(t)-P_{T}(t-1)\right] \\
\quad=\sum_{t^{\prime}}^{t-1} a_{\sigma}\left(t^{\prime}\right)\left[P_{T}(t)-P_{T}(t-1)\right]\end{array}$ \\
\hline Minority game & $-a_{\sigma}(t)[P(t+1)-P(t)]$ \\
\hline$\$$-game & $a_{\sigma}(t-1)[P(t+1)-P(t)]$ \\
\hline Majority game & $a_{\sigma}(t)[P(t+1)-P(t)]$ \\
\hline
\end{tabular}

self-generated price in the model by real Hang Seng Index (HSI) data. For convenience, we call this wealth-based payoff scheme the wealth game. All the transaction price and history of price change for strategy predictions are made with respect to the real data. The data series spans 5045 trading days, from 2 January 1987 to 17 May 2007.

\section{A. Tests with fixed maximum position}

We first consider the case that agents start with zero wealth and have fixed maximum positions $K$. During this period, the agent trades in the market a fictitious stock whose unit price equals the Hang Seng Index. She makes her buy, sell, and hold decisions using the strategies she holds, updating the wealth-based payoffs of her strategies. For comparison, we also consider the update of payoffs using the minority game, the $\$$-game, and the majority game. The payoffs in these other schemes are calculated with the excess demand being replaced by the price change $P(t+1)-P(t)$. A summary for the schemes is given in Table I.

Figure 14 shows the wealth distribution of the four payoff schemes at the end of the trading period. The wealth distribution for the wealth game is distinct from the other schemes in that it has a prominent peak strongly biased towards the direction of high wealth. In contrast, the minority game has a

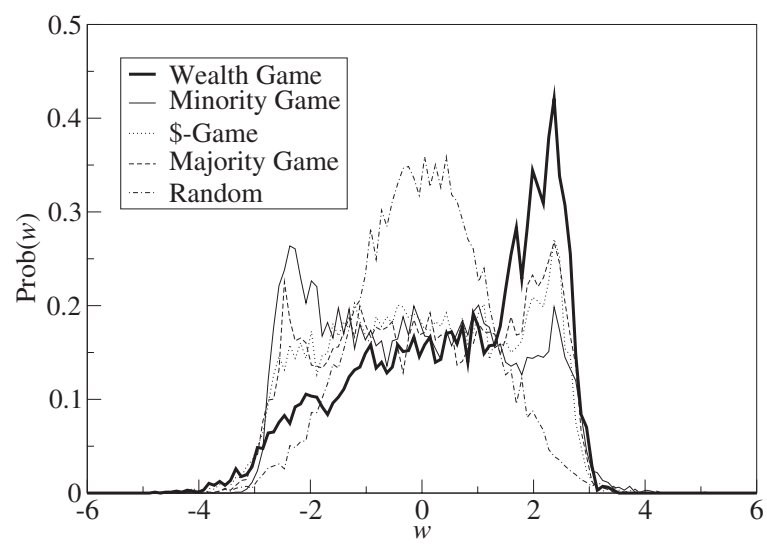

FIG. 14. The final wealth distribution for agents in the wealth game, the minority game, the \$-game, and the majority game, in multiples of the HSI closing price of 17 May 2007. Parameters: $m=2, s=2, K=3, \beta=0.5,10000$ agents for each payoff scheme.
TABLE II. The average wealth and the wealth of the richest agent in the wealth game, the minority game, the \$-game, and the majority game, in multiples of the HSI closing price of 17 May 2007.

\begin{tabular}{lcccc}
\hline \hline Payoff schemes & $\begin{array}{c}\text { Wealth } \\
\text { game }\end{array}$ & $\begin{array}{c}\text { Minority } \\
\text { game }\end{array}$ & \$-game & $\begin{array}{c}\text { Majority } \\
\text { game }\end{array}$ \\
\hline Average wealth & 0.6 & -0.12 & 0.03 & 0.08 \\
Best wealth & 3.31 & 4.51 & 3.35 & 3.38 \\
\hline \hline
\end{tabular}

broad peak biased towards the direction of low wealth. The average wealth of the payoff schemes is summarized in Table II. This shows that the wealth game produces agents with the best average performance.

On the other hand, Table II also shows that the wealth of the richest agent produced by the wealth game is less than those of the other payoff schemes, and much less than that of the minority game, in particular. This indicates that while the wealth game produces good agents on average, it does not encourage the exploration of risky strategies. On the other hand, the minority-seeking nature of the minority game enables the agents to explore unconventional strategies giving rise to unusual success, but this is achieved through sacrificing the performance averaged over the rest of the agents.

\section{B. Tests with wealth-based maximum position}

To make the model even more realistic, we consider the case in which the agents have different maximum allowed positions $K_{i}(t)$ which evolve with time in accordance to their wealth and the current price, such that $K_{i}(t)$ equals the integer part of $\max \left[w_{i}(t) / P(t), 0\right]$. The wealth of every agent is initialized with the same amount of cash wealth $w_{i}(0)$ $=5 P(0)$ to encourage their initial participation in the trading of HSI, that is, their allowed maximum positions are $K_{i}(0)$ $=5$ initially. Compared with the previous case of fixed maximum position, one new feature in this test is that as time goes on, the wealth of some agents decreases below the current price and they are not able to open a new position.

Figure 15 shows the HSI, the wealth of the three best players, five typical players, and the worst player among 10000 players, rescaled by their respective initial values. We make the following observations. (1) It is possible to have agents whose wealth grows faster than the price inflation of HSI. (2) Before the East Asian financial crisis in 1997, the best three players adapt to the booming environment by holding large numbers of stocks (positive positions), capitalizing on the rising trend of the price. However, they are not prepared for the crash. Thus, they all suffer a great loss in the crash as they are still holding high, long positions immediately before the crash. (3) For the typical players, many of them are already lagging behind the price inflation, and thus are not rich enough to hold large numbers of stocks. As a result, they suffer less than the best players from the crash. (4) Some players have their wealth eventually falling below the price. This limits them from opening new positions. Their wealth becomes frozen. 


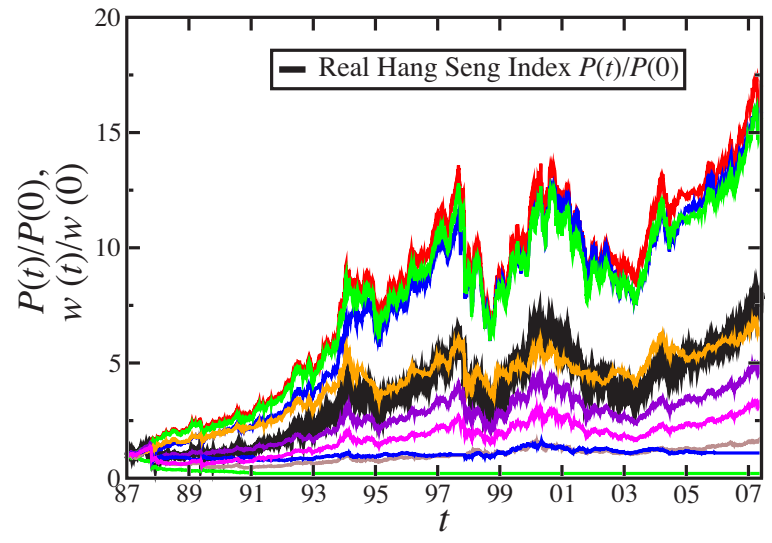

FIG. 15. (Color online) The HSI and the wealth of the three best players, five typical players, and the worst player in the wealth game, all in multiples of the corresponding values on 2 January 1987. Parameters: $m=3, s=2, \beta=0.5$, and $N=10000$.

Figure 16 shows the distribution of final wealth of the agents after trading over 20 years of HSI in this experiment. About $10 \%$ of the agents are gaining agents, that is, with their wealth growing faster than the price inflation of HSI, as indicated by the tail of the distribution to the right of the vertical line in Fig. 16. The wealth of other agents grows slower than the HSI. Peaks near integer values are observed, corresponding to agents whose wealth is clustered around their maximum allowed positions.

The wealth game is compared with other conventional payoff schemes. A control experiment is also done by agents randomly making decisions. Table III shows that among the four payoff schemes, the wealth game has the best average performance, as evident from the average wealth and the fraction of gaining agents. As a benchmark, there are $0.03 \%$ of gaining agents in the case of random choices.

Another advantage of the wealth game can be seen by relaxing the constraint that the strategies held by the agents

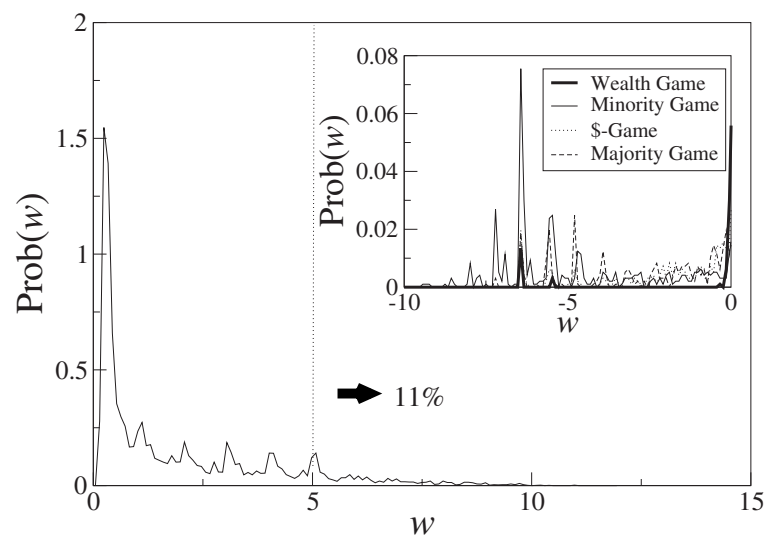

FIG. 16. The distribution of wealth, in multiples of the HSI closing price of 17 May 2007, among the 10000 players in the wealth game with $m=3, s=2, \beta=0.5$. Inset: The distribution of negative wealth, in multiples of the HSI closing price of 17 May 2007, among the 10000 players in the wealth game, the minority game, the \$-game, and the majority game, with $m=3, s=2, \beta$ $=0.5$. The restriction of having at least one buy and one sell decision per strategy is relaxed.
TABLE III. The performance of the agents in the wealth game, the minority game, the \$-game and the majority game, in multiples of the HSI closing price of 17 May 2007.

\begin{tabular}{lcccc}
\hline \hline Payoff schemes & $\begin{array}{c}\text { Wealth } \\
\text { game }\end{array}$ & $\begin{array}{c}\text { Minority } \\
\text { game }\end{array}$ & \$-game & $\begin{array}{c}\text { Majority } \\
\text { game }\end{array}$ \\
\hline Average wealth & 1.85 & 1.47 & 1.40 & 1.31 \\
\% Gaining agents & 10.4 & 8.68 & 6.40 & 5.45 \\
Worst wealth & 0.13 & 0.13 & 0.13 & 0.12 \\
\% Bankruptcy & 0 & 0 & 0 & 0 \\
Best wealth & 11.16 & 17.15 & 11.13 & 10.88 \\
\hline \hline
\end{tabular}

must have at least one buy and one sell decision. This constraint prevents an agent from getting trapped in a long (short) position in a bearish (bullish) market, and hence greatly reduces bankruptcy, that is, negative wealth, as illustrated in Table III by the worst wealth being positive and the bankruptcy rate being 0 .

However, removing this constraint significantly increases the bankruptcy rate. This is especially significant for the minority game, where the bankruptcy rate is highest among the four payoff schemes, and the poorest agents have largely negative wealth, as shown in the inset of Fig. 16. On the other hand, the same figure shows that the bankruptcy rate for the wealth game is much lower, and the poorest agents only have a marginally negative wealth. Indeed, the bankruptcy rates are $0.7 \%, 4.7 \%, 2.6 \%$, and $3.0 \%$ for the wealth, minority, \$-, and majority games, respectively. This advantage of the significantly reduced bankruptcy rate is probably due to the faithfulness of the wealth game payoff in reflecting the wealth of an agent.

On the other hand, the minority game is able to produce a small number of agents with best performances, as shown in Table III. This indicates that the minority game rewards more risky strategies that can result in a small number of successful strategies, a conclusion consistent with the results in the case of fixed maximum position.

At first sight, it does not appear surprising that the agents in the wealth game perform better than other players, since their wealth is updated with the rule of this model. However, the following two tests provide further insights about the behaviors of the different payoff schemes.

First, we test the payoff schemes on the HSI data for a trendy period. We consider the period of recovery from SARS, starting from the bottom on April 25, 2003 to May 17,2007 . The price trend is rather monotonic, making it easy for strategies to adopt. In this case, the wealth game has the best average wealth, and the minority game the worst.

Next, we consider a rugged period from July 15, 1996 to March 21, 2001 (one year before and after the peaks at July 15, 1997 and March 21, 2000, respectively), covering both the East Asian financial crisis and the dot-com bubble. During this period, the general trend of the HSI consists of two rises and two falls, making it rather difficult for the strategies to adopt. In this case, the minority game performs much better than the other payoff schemes, and the wealth game has the poorest average wealth.

In summary, the wealth game performs better than the minority game in trendy periods of HSI, but worse in rugged 
periods. This is probably due to the use of positions $k_{\xi}(t$ $+1)$ rather than the action $a_{\xi}(t)$ in the calculation of the payoff of strategy $\xi$. This gives a stronger history dependence of the wealth game, which favors its perfomance in trendy markets. However, this is probably the same reason that makes it face more difficulties in periods with many trend reversals. Overall, in a time scale as long as 20 years, the price time series of most financial markets have rising trends such as the one in HSI, so that payoff schemes with strong history dependence still have overall advantages.

\section{CONCLUSION}

We have considered a family of models of the financial market in which all parties involved, namely, the agents, the market makers, and the new players, find incentives to participate. This represents a departure from the conventional minority games, whose focus does not reflect the positive sumness of financial markets. The prerequisite of this departure is the direct usage of wealth as both the payoff for evaluating strategies and a measure of the success of individual agents, the latter allowing for the possibility of positive sumness of the market.

The wealth-based payoff scheme is successful in reproducing collective behaviors of the agents resembling those in real markets. Specifically, the price sensitivity $\gamma$ and the market impact $\beta$ are introduced to fine tune, respectively, the transduction from the excess demand to the price dynamics, and the reaction of collective decisions on individuals. In the phase space of $\gamma$ and $\beta$, several behaviors resembling those in real financial markets emerge. In the arbitrageurs' attractor, alternate buy and sell cycles emerge when the market impact is low, analogous to the arbitraging activities of privileged traders in real financial markets.

In the trendsetters' attractor, quasiperiodic cycles of price rises and falls are created by the trendsetters and followed by the fickle agents. This coordinated behavior has a direct correspondence to the roles played by the different kinds of agents in the real market. It demonstrates the importance of using virtual wealth to measure the success of strategies. Since the virtual wealth depends on the history of decisions, it embeds a longer memory in strategy selection, thus inducing a greater sophistication in the collective behavior such as that exhibited in the trendsetters' attractor.

The phases of the arbitrageurs' and trendsetters' attractors are bounded by phase transition lines typical of statistical physics. Their quasiperiodicity demonstrates the robustness of the players' coordinated behavior. On the other hand, while they reproduce agent behaviors plausible in real markets, it seems that their rigid periodicities cannot be reproduced in real markets. It will be interesting to study whether dynamical behaviors of the same nature can be generated by models with more heterogeneity, say, in the memory sizes and maximum positions of the agents.

The phase diagram also consists of the irregular phase, in which the time series of the price consists of intermittent quasiperiodic states connected by abrupt changes. Simple or linear price trends are not observed, making the system dynamically interesting. Fluctuations in the long time scales embody fluctuations in shorter time scales. This is the regime with price time series resembling real markets most.

The phase diagram is rather robust to variations of the model. For example, in the grand-canonical version in which agents with poor strategies are allowed to refrain from playing, we found that the trendsetters' attractor is still sustained by the presence of fickle agents. We have checked that the behavior of the other phases are qualitatively the same in the grand-canonical game.

An interesting question is what parameter range of $\gamma$ and $\beta$ should correspond to the real market. The attraction of agent-based models is their ability to relate an adjustable microscopic mechanism with macroscopic market behavior, thereby giving rise to self-organizing mechanisms for market evolution. Self-organization of markets has been proposed for their states of criticality [7] and efficiency [6]. Here, we propose a possible mechanism for the market to self-organize to the irregular phase resembling the real market.

Suppose the market is in the trendsetters' phase with a low value of $\gamma$. Since the market is positively summed in this phase, the agents are encouraged to act boldly in bidding and asking to maximize their expected wealth gain. This leads to an increase in the price sensitivity. The trend will continue until the market behavior enters the irregular phase. Near the line of zero sum in the irregular phase, the reduced wealth gain of the agents discourages them from increasing their boldness in their bids and asks, and the value of $\gamma$ reaches a steady value. The mechanism is similar to the selforganization of the investment level to an efficient state in [6].

The self-organization of the market impact $\beta$ might be related to the activities of the arbitrageurs. Suppose the market is in the arbitrageurs' phase with a low value of $\beta$. The profitable arbitraging opportunities attract more arbitrageurs to enter the market. Consequently, each individual arbitrageur is no longer as privileged as before, since their decisions will exert market impact on each other. This results in an increase in the market impact parameter. The trend will continue until the market behavior enters the irregular phase. In practice, individual agents experience different market impacts due to their different reaction times and transaction volumes. Models with an individualized market impact should be studied in the future to further clarify this issue of self-organization.

Returning to the issue of participation incentives, the phase space has a large region of positive sumness. This positive sumness provides incentives for the agents to play in the market. In the absence of new players joining the market, their wealth comes from the market makers who play the role of balancing the supply and demand of the market.

The participation incentives of the market makers come from the bid-ask spread during their transactions with the players. We found that both the fixed transaction cost and fixed transaction rate cannot ensure a stable balance of wealth between the agents and the market makers. Rather, an adaptive transaction rate avoids this problem.

The picture of participation incentives is completed by considering open markets, in which underperforming agents are replaced by new agents. This converts the zero-sum game of the agents and market makers to one with a positive sum. 
The participation incentive of the new players may arise from their chances to learn the strategies of the more successful players. We found that these agents have survival probabilities matching those of random evolution, provided that the evolution is sufficiently slow.

Finally, we have tested the wealth-based payoff scheme by playing the wealth game on HSI data over 20 years, and compared its performance with the minority game, the $\$$-game, and the majority game. We found that the wealth game produces agents with much better average performance than other games, and significantly reduces the bankruptcy rate for agents holding poor strategies. This is probably due to the faithfulness of the payoff scheme in reflecting the wealth associated with a strategy. Further tests on other markets are needed to experiment the applicability of wealthbased payoff schemes and will be reported elsewhere.

On the other hand, the minority game is able to produce a small number of extremely rich agents, whose wealth is better than the richest agents produced by other payoff schemes. This indicates that while the wealth game produces good agents on average, it does not reward the exploration of risky strategies, whereas the minority-seeking nature of the minority game enables the agents to explore unconventional strategies giving rise to unusual success, but this is achieved through sacrificing the performance averaged over the rest of the agents.

The strengths and weaknesses of the different payoff schemes are also revealed in their performances in trendy and rugged periods. The wealth game performs well when history can provide a guide, but meets difficulties in markets with many trend reversals.
These performance characteristics of the wealth-based payoff scheme can be traced to the different ways virtual scores are updated in the payoff schemes. Among the many differences between the wealth game and the minority game, the most essential one is that the position $k_{\sigma}(t-1)$ replaces the action $a_{\sigma}(t)$ in the calculation of the virtual score in Table I. In the wealth game, agents assess their gains by their positions rather than one-step decisions. Since the position of a series of actions of a strategy is equal to its historical sum, position-dependent assessment schemes embed a longer memory scale in the decision making process of agents than those in the minority game.

Interesting issues arising from this comparative study remain. First, wealth-based payoff schemes are able to explain the behavior of good investors statistically, but since the extremely successful investors are produced by the minority game rather than the wealth game, it is possible that the behavior of the group of rich agents and the few extremely rich agents have to be modeled differently, especially with respect to the readiness to take risks. Second, it would be interesting to formulate integrated payoff schemes, so that the agents can both learn from history during trendy periods, and be ready to explore risky but potentially successful strategies during risky periods.

\section{ACKNOWLEDGMENTS}

We thank David Sherrington and Andrea de Martino for meaningful discussions. This work is partially supported by the Research Grant Council of Hong Kong (Grants No. HKUST603606 and No. HKUST603607).
[1] D. Challet, M. Marsili, and Y.-C. Zhang, Minority Games (Oxford University Press, Oxford, UK, 2005).

[2] D. Challet and Y.-C. Zhang, Physica A 246, 407 (1997).

[3] R. Savit, R. Manuca, and R. Riolo, Phys. Rev. Lett. 82, 2203 (1999).

[4] R. Jefferies, M. L. Hart, P. M. Hui, and N. F. Johnson, Eur. Phys. J. B 20, 493 (2001).

[5] J. V. Andersen and D. Sornette, Eur. Phys. J. B 31, 141 (2003).

[6] I. Giardina and J.-P. Bouchaud, Eur. Phys. J. B 31, 421 (2003).

[7] D. Challet, A. Chessa, M. Marsili, and Y.-C. Zhang, Quant. Finance 1, 168 (2001).

[8] F. Slanina and Y.-C. Zhang, Physica A 272, 257 (1999).

[9] D. Challet, M. Marsili, and Y.-C. Zhang, Physica A 276, 284 (2000).

[10] M. Marsili, Physica A 299, 93 (2001).

[11] D. Challet, J. Econ. Dyn. Control 32, 85 (2008).

[12] F. Ren and Y.-C. Zhang, e-print arXiv:0801.3560.

[13] M. Marsili, D. Challet, and R. Zecchina, Physica A 280, 522
(2000).

[14] D. Challet, M. Marsili, and R. Zecchina, Phys. Rev. Lett. 84, 1824 (2000).

[15] J. A. F. Heimel and A. C. C. Coolen, Phys. Rev. E 63, 056121 (2001).

[16] Y.-C. Zhang, Physica A 269, 30 (1999).

[17] C. H. Yeung and K. Y. M. Wong, Europhys. Lett. 75, 357 (2006).

[18] D. Challet and M. Marsili, Phys. Rev. E 60, R6271 (1999).

[19] K. Y. Michael Wong, S. W. Lim, and Z. Gao, Phys. Rev. E 70, 025103(R) (2004).

[20] K. Y. Michael Wong, S. W. Lim, and Z. Gao, Phys. Rev. E 71, 066103 (2005).

[21] D. Challet, M. Marsili, and Y.-C. Zhang, Physica A 294, 514 (2001).

[22] Y. Liu, P. Gopikrishnan, P. Cizeau, M. Meyer, C. K. Peng, and H. E. Stanley, Phys. Rev. E 60, 1390 (1999). 